\title{
PROCAMALLANUS BRASILEIROS - PARTE I (NEMATODA, CAMALLANOIDEA) ${ }^{1}$
}

\author{
R. MAGALhĀES PINTO*, SUELI P. DE FABIO*, DELY NORONHA* \\ \& F. J. TAYT-SON ROLAS** \\ Instituto Oswaldo Cruz, Rio de Janeiro, Guanabara, Brasil \\ (Com 5 estampas)
}

\begin{abstract}
SUMÁRIO: Nessa primeira parte do trabalho referente ao estudo dos Procamallanus brasileiros, os autores propõem que Spirocamallanus Olsen, 1952, seja aceito apenas como um bom subgênero, baseando-se exclusivamente no aspecto da cápsula bucal. Assim, Spirocamallanus identifica os camalanídeos de cápsula bucal com lâminas espiraladas ou ou tras estruturas semelhantes, enquanto que Procamallanus se aplicaria aos de cápsula bucal lisa. Quanto às demais subdivisões existentes para o gênero, apoiadas em aspecto e quantidade de espículos, são consideradas resultado de um critério bastante arbitrário para conservarem-se válidas, visto serem os espículos caracteres sujeitos a diversas interpretações por parte dos especialistas, devido à fragilidade que os toma tão sujeitos a modificações no grupo dos camalanídeos. Três espécies são aqui tratadas: Procamallanus (Spirocamallanus) rarus Travassos, Artigas \& Pereira, 1928, Procamallanus (Spirocamallanus) pimelodus, e Procamallanus (Spirocamallanus) intermedius. As duas últimas são propostas como novas. Procamallanus (S.) pimelodus é comparada a Procamallanus (Spirocamallanus) olseni Campana-Rouget \& Razahihelissoa, 1965, dela se distinguindo, principalmente, por apresentar o rebordo da asa caudal franjado e fortemente musculoso. Procamallanus (S.) intermedius aproxima-se unicamente de Procamallanus (S.) rarus, por apresentar o espículo maior com bifurcação distal e dela se afasta pelo maior número e menor espessura das lâminas espiraladas na cápsula bucal. Para Procamallanus (S.) rarus é apresentada a descrição da fêmea, a redescrição do macho, salientando-se pela primeira vez no gênero, o aspecto singular do espículo maior, aspecto esse também observado ao se lidar com Procamallanus (S.) intermedius, depois de se ter tido acesso ao exemplar tipo e único de Procamallanus (S.) rarus. Foram examinadas 31 amostras de material que faz parte da Coleç̧ão Helmintológica do Insti tu to Oswaldo Cruz.
\end{abstract}

gênero Procamallanus, criado por Baylis em 1923, tem, desde então, dado origem a uma série de discussões por parte da maioria dos sistematas que vem tentando grupar os helmintos propostos para Procamallanus, de modo que novos arranjos possam englobar a grande variação de aspecto com que esses nematódeos se apresentam. Há de se notar que as tentativas para obter uma estrutura razoável para o gênero, admitindo subdivisões, vem da necessidade de facilitar o estudo desse grupo. Partindo desse princípio, os caracteres que têm servido de base para que vánias soluções sejam apresentadas, são a cápsula bucal bastante peculiar e os espriculos. No presente trabalho, a meta principal não é a de se fazer uma revisão de gênero, mas sim, a de ampliar o conhecimento dos Procamallanus brasileiros. Nosso estudo será realizado em etapas, vis to a existência de farto material depositado em nossas coleções. Entretanto, nessa primeira parte, queremos, também, emitir nosso conceito atual sobre o gênero, procurando mais uma vez, contribuir no sentido de racionalizar sua organização.

1 Entregue para publicação em 25 de janeiro de 1974.

Trabalho do Laboratório de Helmintologia do Departamento de Zoologia Médica do Instituto Oswaldo Cruz, realizado, em parte, com o auxílio do CNPq.

* Bolsistas do CNPq.

** Bolsista do Instituto Oswaldo Cruz. 


\section{MATERIAL E MÉTODOS}

Os helmintos estudados estavam conservados em líquido de Railliet \& Henry. Foram diafanizados em ácido acético, fenol e creosoto de faia e montados em bálsamo do Canadá. Como os Procamallanus têm pouquíssima afinidade com os corantes usuais, não lançamos mão deles, obtendo, todavia, resultados plenamente satisfatórios. Entretanto, devemos assinalar os acidentes durante a manipulação do material que sempre se apresentou muito delicado, ocasionando rupturas e macerações ou por excessiva fragilidade ou pela conservação precária, visto ser a maioria das necrópsias efetuada em datas remotas. Os espículos de Procamallanus (S.) rarus foram melhor observados quando submetidos à ação de luz polarizada. As fotografias das cápsulas bucais foram obtidas em microscópio Olympus e as figuras originais desenhadas em câmara-clara Ernst Leitz.

Os estudos são baseados em 31 amostras, sendo 27 parasitas de Pimelodus clarias (L.), 3 de bagre (inde terminado) e 1 de Pimelodella lateristriga (Müll. \& Trosch).

\section{RESULTADOS}

Em trabalhos anteriores; no Brasil, já haviam sido descritos os seguintes nematódeos pertencentes ao gênero Procamallanus Baylis, 1923:

1 - Procamallanus (Spirocamallanus) iheringi Travassos, Artigas \& Pereira, 1928.

Sinonímia: Procamallanus iheringi Travassos, Artigas \& Pereira, 1928: 21, est. 6. figs. 56-61; Spirocamallanus iheringi Olsen, 1962: 196, Procamallanus (Procamallanus) iheringi: Ali, 1960: 137.

Habitat: Intestino e divertículos pilóricos de Salminus hilarii (Cuv. \& Val) (Tabarana), Hoplias sp. (Trairão), Tetragonopterus sp. (Lambari), Leporinus sp. (Piaba) Leporinus sp. (Piapara), Anostomatinae sp. (Taguara), Anostomatinae sp. (Ferreira).

Distribuição geográfica: Emas, Pirassununga, S. Paulo. Referências: 2,8 e 17 .

2 - Procamallanus (Spirocamallanus) inopinatus Travassos, Artigas \& Pereira, 1928.

Sinonímia: Procamallanus inopinatus Travassos, Artigas \& Pereira, 1928: 21, est. 6, figs.65-70; Spirocamallanus inopinatus Olsen, 1952: 196; Procamalla- nus (Isospiculus) inopinatus: Ali, 1960: 136; Procamallanus fariasi: Pinto \& Noronha, 1972: 105-108, 10 figs.

Habitat: Intestino de Leporinus sp. (Piapara), leporinus sp. (Piaba), "Cangati", Pygocentrus sp.

Distribuição geográfica: Emas, Pirassununga, S. Paulo; Harmonia, Alfenas, Minas Gerais, Cruzeta, Rio Grande do Norte.

Referências: $2,8,12$ e 17 .

3 - Procamallanus (Spirocamallanus) rarus Travassos, Artigas \& Pereira, 1928.

Sinonímia: Procamallanus rarus Travassos, Artigas \& Pereira, 1928: 22, est. 6, figs. 62-64; Spirocamallanus rarus Olsen, 1952: 196; Procamallanus (Procamallanus) rarus: Ali, 1960: 137.

Habitat: Intestino de Pimelodella lateristriga (Müll. \& Trosch) Rhynodoras dorbignyi Kröyer (Mandi-serra).

Distribuição geográfica: limas, Pirassununga, S. Paulo. Referências: 2,8 e 17 .

4 - Procamallanus (Spirocamallanus) amarali Vaz \& Percira, 1934.

Sinonímia:

Procamallanus amarali Vaz \& Pereira, 1934: 96-97, figs. 21-23; Spirocamallanus amarali Olsen, 1962: 196; Procamallanus (Procamallanus) amarali: Ali, 1960: 137.

Habitat: Intestino delgado de Leporinus sp. (Piaba de abas claras).

Distribuiçāo geográfica: Rio Tictê (Salto) lstado de S. Paulo.

Referências: 2,8 e 18.

5 - Procamallanus (Spirocamallanus) hilarii Vaz \& Perira, 1934.

Sinonímia: Procamallanus hilarii Vaz \& Pereira, 1934: 98-99, figs. 24-28; Spirocamallanus hilarii Olsen, 1952: 196: Procamallanus (Procamallanus) hilarit: Ali, 1960: 138.

Habitat: Intestino delgado de Salminus hilarii (Tabarana)

Distribuição geográfica: Represa de Santo Amaro, Estado de S. Paulo.

Referências: 2,8 e 18 . 
6 - Procamallanus (Spirocamallanus) wrighti Pereira, 1935.

Sinonímia: Procamallanus wrighti Pereira, 1935: 58-60, figs. 17-21; Spirocamallanus wrighti Olsen, 1952: 197; Procamallanus (Isospiculus) wrighti: Ali, 1960: 136.

Habitat: Intestino de Astyanax sp., Hoplias malabaricus, Leperinus sp., Pygocentrus sp.

Distribuição geográfica: Areia, Patos, Paraíba; Cruzeta, Caicó, Rio Grande do Norte.

Referências: 2,8 e 10 .

7 - Procamallanus (Spirocamallanus) barroslimai Pereira, 1935.

Sinonímia: Procamallanus barroslimai Pereira, 1935: 60-61, figs. 22-23; Spirocamallanus barroslimai Olsen, 1952: 196; Procamallanus (Procamallanus) barroslimai: Ali, 1960: 138.

Habitat: Intestino de "Sardinha".

Distribuição geográfica: Cruzeta, Rio Grande do Norte.

Referências: 2,8 e 10 .

8 - Procamallanus (Spirocamallanus) cearensis Pereira, Dias \& Azevedo, 1936.

Sinonímia: Procamallanus cearensis Pereira, Dias \& Azevedo, 1936: 209-226, 8 figs., ests. 30-33; Spirocamallanus cearensis Olsen, 1952:197; Procamallanus (Isospiculus) cearensis: Ali, 1960: 136.

Habitat: $\quad$ Adultos: Intes tino delgado de Astya. nax bimaculatus vittatus Castelnau, 1855; Larvas de 1.0 e 2.0 estádio: Cavidade geral de Diaptomus cearensis Wright, 1936 e $D$. azevedoi Wright, 1935; Larvas de $2 .^{\circ}$ e $3 .^{\circ}$ estádios: Alevinos de Curimatus elegans Steindachner.

Distribuição geográfica: Lagoas de Tauapé, Soure e Porangaba, Fortaleza, Ceará.

Referências: 2,8 e 11 .

9 - Procamallanus (Spirocamallanus) probus Pinto \& Fernandes, 1972.

Sinonímia: Procamallanus probus Pinto \& Fernandes, 1972: 133-136, 6 figs.
Habitat: Intestino de Brycon brevicaudatus Gunther.

Distribuição geográfica: Rio Machado, Porto Velho, Rondônia.

Referência: 13.

10 - Procamallanus (Spirocamallanus) macaensis Vicente \& Santos, 1972.

Sinonímia: Procamallanus macaensis Vicente \& Santos, 1972: 145-147, 5 figs.

Habitat: Intestino de Menticirrus americanus (L.).

Distribuição geográfica: Macaé, Estado do Rio de Janeiro.

Referência: 19.

No presente trabalho, estudamos os seguintes helmintos:

Procamallanus (Spirocamallanus) rarus Travassos, Artigas \& Pereira, 1928.

(Est. I, II, V figs. 5, 8, 18, 19, 20, 21 e 22)

\section{DESCRIÇÃO:}

$\begin{array}{llrlr}\text { Comprimento: } & \text { Machos } & -9,24 & \text { a } & 13,53 \mathrm{~mm} \\ & \text { Fêmeas } & -11,05 & \text { a } & 25,62 \mathrm{~mm} \\ \text { Largura: } & \text { Machos } & -0,18 & \text { a } & 0,20 \mathrm{~mm} \\ & \text { Fêmeas } & -0,20 & \text { a } & 0,34 \mathrm{~mm}\end{array}$

Nematódeos de coloração amarelo-pálido quando conservados. Corpo fusiforme, delgado, com cutícula estriada transversalmente. Poro excretor não evidenciado. Anel nervoso distando 0,16 a $0,21 \mathrm{~mm}$ da extremidade anterior nos machos e 0,22 a $0,23 \mathrm{~mm}$ nas fêmeas. Boca circular, dando entrada à cápsula bucal que é ampla, bastante quitinizada, com lâminas espiraladas muito salientes, em número de 3 ou 4, medindo 0,084 a $0,10 \mathrm{~mm}$ de comprimento nos machos e 0,084 a $0,18 \mathrm{~mm}$ por 0,046 a $0,11 \mathrm{~mm}$ de largura nas fêmeas. Esôfago muscular com 0,50 a $0,81 \mathrm{~mm}$ de comprimento por 0,056 a $0,070 \mathrm{~mm}$ de largura nos machos e 0,65 a $1,39 \mathrm{~mm}$ por $0,070 \mathrm{~mm}$ a $0,084 \mathrm{~mm}$ nas fêmeas. Esôfago glandular (ventrículo) medindo $0,80 \mathrm{~mm}$ de comprimento por 0,042 a $0,084 \mathrm{~mm}$ de largura nos machos e 0,66 a $1,64 \mathrm{~mm}$ por 0,042 a $0,084 \mathrm{~mm}$ nas fêmeas.

Fêmeas vivíparas, didelfas, anfidelfas. Vulva mediana. Reto medindo $0,24 \mathrm{~mm}$ de comprimento. Ânus distando $0,15 \mathrm{~mm}$ da extremidade posterior. Espinho caudal ausente.

Machos com extremidade caudal curvada ventralmente, com asas caudais relativamente estritas e 8 pares de papilas pedunculadas, distribuídas da seguinte 
maneira: 4 pares pré-anais e 4 pares pós-anais. Gubernáculo ausente. Espículos desiguais e dissemelhantes, tendo, o maior, aspecto bastante peculiar (figs. 19, 20 e 22). Este, levando-se em consideração sua morfologia, foi dividido em zonas (figs. 19 e 20) e as medidas foram feitas em direção ao comprimento do espículo. Assim temos: $\mathrm{AB}=0,63$ a $0,64 \mathrm{~mm} ; \mathrm{AC}=0,48$ a $0,52 \mathrm{~mm} ; C B=0,090$ a $0,096 \mathrm{~mm} ; C D=0,090$ a $0,114 \mathrm{~mm}$. O menor mede 0,22 a $0,28 \mathrm{~mm}$ de comprimento. Ānus distando $0,10 \mathrm{~mm}$ da extremidade caudal.

Habitat: Intestino de Bagre (indeterminado) Distribuição geográfica: Rio Amazonas, Maicuru, Pará.

Material estudado depositado na Coleção Helmintológica do Instituto Oswaldo Cruz sob os n.os 31026 a-c, 31027 a-b, 31028 a-c e 31029 (tipo).

COMENTÁRIO: Travassos et col. (1928) ao criarem Procamallanus (Spirocamallanus) rairus, de um único exemplar macho, talvez não tenham dado uma in terpretação adequada ao descrever e figurar o espículo maior. Como tivemos acesso ao tipo, depositado na Coleção Helmintológica do Instituto Oswaldo Cruz, verificamos que o espículo é bastante característico, idêntico ao que se nos apresentou ao lidarmos com amostras parasitas de peixe dulcícola, um bagre, indeterminado. Desta forma, identificamos os nematódeos a Procamallanus. (S.) rarus, tomando conhecida a fêmea da espécie e redescrevemos o macho, assinalando pela primeira vez em Procamallanus o aspecto curioso do espículo maior e ampliamos sua distribuição geográfica, visto a espécie haver sido descrita de material proveniente de Emas, Pirassununga, S. Paulo, parasitando Pimelodella lateristriga (Müll. \& Trosch.) e Rhynodoras dorbignyi Kröyer, Mandi-chorão e Mandi-serra, respectivamente, ambos dulcícolas. Apesar da referência do último hospedador para Procamallanus (S.) rarus, julgamos conveniente considerar apenas Pimelodella lateristriga como tal, pois Travassos et col. propuseram a espécie baseando-se em apenas 1 exemplar. Após consultarmos nossos arquivos, tivemos nossas hipóteses confirmadas, pois Pimelodella lateristriga é o único hospedador referido para Procamallanus (S.) rarus. O tipo por nós es tudado estava conservadó em formol acético sob o n. 0 5.930. O material foi montado em lâmina e conservado em bálsamo sob o n.o 31.029 .

Referências: 17,20 e 21.

Procamallanus (Spirocamallanus) pimelodus sp. $\mathrm{n}$.

(Ests. I, II, III figs. 1, 2, 3, 7, 9, 10, 11, 12 e 13)

\section{DESCRIÇĀO:}

Comprimento: Machos - 7,63 a 11,34 mm
Fêmeas - 11,76 a $23,17 \mathrm{~mm}$

Largura: $\quad$ Machos - 0,15 a $0,22 \mathrm{~mm}$

Fêmeas - 0,17 a $0,23 \mathrm{~mm}$

Nematódeos de coloração variando do amarelo pálido ao marrom-escuro quando conservados. Corpo fusiforme, delgado, com cutícula estriada transversalmente. Poro excretor situado de 0,24 a $0,27 \mathrm{~mm}$ da extremidade anterior nas fêmeas e não evidenciado nos machos. Anel nervoso distando $0,25 \mathrm{~mm}$ da extremidade anterior em ambos os sexos. Boca circular, dando entrada à cápsula bucal que é ampla, bastante quitjnizada, com lâminas espiraladas muito salientes. Mede 0,055 a $0,066 \mathrm{~mm}$ de comprimento por 0,040 a $0,052 \mathrm{~mm}$ de largura nos machos e 0,049 a 0,055 por $0,037 \mathrm{~mm}$ nas fêmeas. O número das lâminas varia de 6 a $7 \mathrm{em}$ ambos os sexos. Algumas são incompletas, alcançando cerca de $2 / 3$ da largura total da cápsula. Esôfago muscular me dindo $0,47 \mathrm{~mm}$ de comprimento por $0,057 \mathrm{~mm}$ de largura nos machos e 0,34 a 0,50 por $0,057 \mathrm{~mm}$ nas fêmeas. Esôfago glandular (ventrículo) medindo $0,80 \mathrm{~mm}$ de comprimento por $0,057 \mathrm{~mm}$ de largura nos machos e 0,51 a $0,57 \mathrm{~mm}$ por 0,046 a $0,057 \mathrm{~mm}$ nas fêmeas.

Fêmeas vivíparas, didelfas, anfidelfas, com ovejetor fortemente musculoso, com cerca de $0,57 \mathrm{~mm}$ de comprimento. Vulva distando 3,57 a 4,06 $\mathrm{mm}$ da extremidade anterior. Ovejetor longo e sinuoso que se dirige primeiramente para trás, e após apresentar curva em $U$, desviando-se lateralmente, bifurca-se, dando origem aos 2 ramos uterinos que são opostos. 0 ramo ascendente termina um pouco abaixo do esôfago glandular (ventrículo) a uma distância de aproximadamente $1,4 \mathrm{~mm}$ da extremidade anterior. Apresenta em sua porção terminal forma globosa com apêndice recurvado. $O$ ramo descendente é pouco desenvolvido e termina de modo indistinto. $\mathrm{Na}$ maioria das vezes o útero se encontra repleto de larvas, dificultando a observação das estru turas internas. Ânus distando $0,08 \mathrm{~mm}$ da extremidade posterior. Reto com 0,029 a $0,031 \mathrm{~mm}$ de comprimento. Espinho caudal presente, com $0,019 \mathrm{~mm}$ de comprimento.

Machos com extremidade caudal curvada ventralmente, apresentando asas caudais fortemente musculosas que se juntam na superfície ventral. Papilas caudais sésseis, em número de 6 pares, distribuídos da seguinte maneira: 3 pares pré-anais e 3 pares pós-anais (fig. 9). Gubernáculo ausente. Espículos desiguais, pouco quitinizados. 0 maior mede 0,39 a $0,48 \mathrm{~mm}$ de comprimento e o menor 0,16 a $0,24 \mathrm{~mm}$ de comprimento. Ânus distando $0,10 \mathrm{~mm}$ da extremidade caudal.

Habitat: Intestino de Pimelodus clarias (L.)

Distribuição geográfica: Porto Esperança, Mato Grosso e Pirassununga, S. Paulo.

Holótipo macho 30993 a, holótipo fêmea 30.999 a e 
parátipos $30.989 \mathrm{a}-\mathrm{b}, 30.990,30.991,30.992 \mathrm{a}-\mathrm{b}$, $30.993 \mathrm{~b}-\mathrm{i}, 30.994,30.995 \mathrm{a}-\mathrm{b}, 30.996,30.997 \mathrm{a}-\mathrm{b}$, 30.998 a-c, 30.999 b-f, 31.000 a-c, $31.001,31.002$ a-b, 31.003 a-b, $31.004 \mathrm{a}-\mathrm{c}, 31.005 \mathrm{a}-\mathrm{c}, 31.006 \mathrm{a}-\mathrm{d}, 31.007$, $31.008 \mathrm{a}-\mathrm{b}, 31.009 \mathrm{a}-\mathrm{c}, 31.010,31.011$, estudados, depositados na Coleção Helmintológica do Institu to Oswaldo Cruz.

DISCUSSÃO: A espécie agora descrita mais se aproxima de Procamallanus (S.) olseni CampanaRouget \& Razahihelissoa, 1965, pelo tamanho dos espículos, asas caudais bem desenvolvidas que se juntam na superfície ventral, e dela se afasta, pelo menor número, disposição e aspecto das papilas caudais e número de lâminas na cápsula bucal. Além disso, em Procamallanus $(S$.$) pimelodus sp. n., as asas caudais$ apresentam rebordos franjados com forte musculatura, que lhe dão aspecto característico (figs. 9 e 11 ). Devido a esse caráter, as papilas são pouco evidenciadas quando o helminto não é visto de frente. Procamallanus $(S$.) olseni é parasito de peixes marinhos (Echeneis naucrates, L., Lutjanus duodecimlineatus e Lutjanus sp.) provenientes de Nossi - Bé, África, enquanto que o hospedador da nova espécie é dulcícola.

Referências: $3,5,6,10,11,12,13,17,18,19,20 \mathrm{e}$ 21.

Procamallanus (Spirocamallanus) intermedius sp. $\mathrm{n}$.

(Ests. I, II, IV figs. 4, 6, 14, 15, 16 e 17)

\section{DESCRIÇÃO:}

$\begin{array}{ll}\text { Comprimento: } & \text { Machos - 8,02 a } 10,29 \mathrm{~mm} \\ & \text { Fêmeas - 11,30 a } 19,67 \mathrm{~mm} \\ \text { Largura: } & \text { Machos - 0,15 a } 0,23 \mathrm{~mm} \\ & \text { Fêmeas - 0,17 a } 0,31 \mathrm{~mm}\end{array}$

Nematódeos de coloração marrom-escuro, quando conservados. Corpo fusiforme, delgado, com cutícula estriada transversalmente. Poro excretor distando 0,24 a $0,26 \mathrm{~mm}$ da extremidade anterior nos machos e 0,31 a $0,34 \mathrm{~mm}$ nas fêmeas. Anel nervoso distando $0,23 \mathrm{~mm}$ da extremidade anterior, em ambos os sexos. Boca circular, dando entrada à cápsula bucal que é ampla, bastante quitinizada, com lâminas espiraladas muito salientes, em número de 6 nos machos e 9 nas fêmeas, medindo $0,072 \mathrm{~mm}$ de comprimento por $0,014 \mathrm{~mm}$ de largura nos machos e 0,072 a 0,086 por 0,014 a $0,072 \mathrm{~mm}$ nas fêmeas. Esôfago muscular com 0,53 a $0,56 \mathrm{~mm}$ de comprimento por $0,07 \mathrm{~mm}$ de largura nos machos e 0,60 a 0,72 por 0,07 a $0,10 \mathrm{~mm}$ nas fêmeas. Esôfago glandular (ventrículo) medindo 0,70 a $0,84 \mathrm{~mm}$ de comprimento por 0,01 a $0,08 \mathrm{~mm}$ de largura nos machos e 0,70 a 0,99 por 0,07 a $0,10 \mathrm{~mm}$ nas fêmeas.

Fêmeas vivíparas, didelfas, anfidelfas. Vulva media- na. Reto com 0,11 a $0,14 \mathrm{~mm}$ de comprimento. Ânus distando 0,10 a $0,14 \mathrm{~mm}$ da extremidade posterior. Espinho caudal ausente.

Machos com extremidade caudal curvada ventralmente, com asas caudais relativamente desenvolvidas e 8 pares de papilas pedunculadas, distribuídas da seguinte maneira: 2 pares pré-anais, 1 par ad-anal e 5 pares pós-anais. Gubernáculo ausente. Espículos desiguais e dessemelhantes. O maior é bifurcado na região distal e foi dividido em zonas (figs. 16 e 17) sendo as medidas feitas em direção ao comprimento do espículo. Assim, temos, $A B=0,63 \mathrm{~mm} ; A C=0,56 \mathrm{~mm}$; $\mathrm{CB}=0,072 \mathrm{~mm} ; \mathrm{CD}=0,072 \mathrm{~mm}$. O menor mede 0,24 a $0,28 \mathrm{~mm}$ de comprimento. Ânus distando $0,14 \mathrm{~mm}$ da extremidade caudal.

Habitat: Intestino de Pimelodus clarias (L.)

Distribuição geográfica: Porto Cabral, Rio Paraná, S. Paulo.

Holótipo macho $31.022 \mathrm{a}$, holótipo fêmea $31.023 \mathrm{a}$ e parátipos $31.022 \mathrm{~b}-\mathrm{h}, 31.023 \mathrm{~b}-\mathrm{c}, 31.024,31.025 \mathrm{a}-\mathrm{b}$, estudados, depositados na Coleção Helmintológica do Instituto Oswaldo Cruz.

DISCUSSÃO: Procamallanus (S.) intermedius sp. n. aproxima-se unicamente de Procamallanus $(S$. ) rarus Travassos, Artigas \& Pereira, 1928, pelo aspecto do espículo, e dela se afasta pelo número e espessura das lâminas espiraladas da cápsula bucal, que, na nova espécie são mais numerosas e delgadas.

Referências: $3,5,6,10,11,12,13,17,18,19,20$ e 21.

\section{CONCLUSÕES E DISCUSSÃO}

Como a cápsula bucal e os espículos são os caracteres morfológicos que têm dado origem a controvérsias quanto à sua validade no diagnóstico genérico, subgenérico e específico nesse grupo, iremos nos ater a esses dois aspectos, com o propósito de bem orientamos publicaçð̃es futuras. Com relação à estrutura da cápsula bucal, ratificamos Pinto \& Noronha (1972), não se justificando um desdobramento do gênero, levando-se em consideração a existência ou falta de estruturas formadoras da cápsula. A presença das lâminas espiraladas realmente nos induz a uma separação imediata quando comparamos nematódeos que as possuem, com aqueles de cápsula bucal lisa. Até hoje, porém, várias hipóteses foram lançadas, sem, entretanto, ter se 
conseguido avaliar até que ponto o aparecimento dessas estruturas esteja ligado a fatores genéticos e evolutivos. Referindo-nos aos espículos, discordamos de Ali $(1956,1960)$ quando da proposição dos subgêneros para Procamallanus Baylis, 1923, levando em consideração esse caráter, pois, conforme pudemos observar, os espículos possuem uma quitinização muito fraca, que não permite um diagnóstico preciso. Campagna-Rouget \& Therezien (1965) ao criarem Procamallanus (Procamallanus) armatus, referem a presença de um só espículo, mas consideram: "L'étude d'un seul espécimen mâle ne nous permet malheuresement pas de preciser si l'absence de l'espicule gauche est un caractére constant: Ce dernier, souvent peu chitinisé, peut en effet manquer ou tout au moins, rester invisible ....." "Nous ne pouvons donc considérer la présence d'un seul espicule comme un bon caractére espécifique". Sood, (1967) concordando com diversos autores, afirma: "The author...... also follows Campana-Rouget (1961), Pande, Bhatia and Rai (1963), Fernando e Furtado (1963) and Agrawal (1966) in the suppression of the subgenera of the genus Procamallanus on the basis of differences in agreement with these authors because the spicules are weakly chitinised in camallanids and are therefore of little systematic importance." Desta forma, nosso conceito atual sobre o gênero Procamallanus Baylis, 1923, é somente baseado no aspecto da cápsula bucal. Considerando Spirocamallanus Olsen, 1952, um bom subgênero, teremos:

1 - Procamallanus (Spirocamallanus) quando apresentarem lâminas espiraladas ou quaisquer outras estruturas semelhan tes na cápsula bucal.

\section{2 - Procamallanus (Procamallanus) quando apresentarem cápsula bucal lisa.}

\section{SUMMARY}

Brazilian "Procamallanus"

(Nematoda, Camallanoidea) Part I.
By introducing this work on brazilian nematodes under Procamallanus Baylis, 1923, the authors propose that Spirocamallanus Olsen, 1952 , must be regarded only as a good subgenus based on the aspect of the buccal capsule. For this reason, Spirocamallanus should identify those worms bearing spiral thickenings, as well as similar structures inside their buccal cavity while Procamallanus may be applied every time the nematodes present a completely smooth capsule, lacking the above mentioned structures. Regarding to the early propositions in order to subdivide the genus using number and shape of the spicules as differential characters, the authors fully disagree with this point of view, which is rather a poor criterion, for the spicules are weakly chitinised in this group and for this reason they do not present enough strutural uniformity to be of great systematic significance. The authors deal here with three species: Procamallanus (Spirocamallanus) rarus Travassos, Artigas \& Pereira, 1928, Procamallanus (Spirocamallanus) pimelodus and Procamallanus (Spirocamallanus) intermedius. The last two are proposed as new species. Procamallanus ( $S$. ) pimelodus is related to Procamallanus (Spirocamallanus) olseni CampanaRouget \& Razahihelissoa, 1965 and differs from it mainly by its caudal alae which shows a strongly muscular fringed rim. Procamallanus (S.) intermedius only comes nearer to Procamallanus $(S$.) rarus by possessing the bigger spicule with distal bifurcation and differs from it by the aspect of the buccal capsule which has more and thinner spiral thickenings. Of Procamallanus $(S$. ) rarus, the 'female is described, a redescription of the male is presented and the very peculiar aspect of the bigger spicule is pointed out for the first time in the genus. This was also observed when dealing with Procamallanus $(S$. ) intermedius, after examining the type of Procamallanus (S.) rarus. In this first part of the work the authors handled 31 samples of nematodes as follows: From intestines of Pimelodus clarias (L.)(27); Catfish (indeterminate)(3) and Pimelodella lateristriga (Müll. \& Trosch.)(1). 


\section{REFERÊNCIAS BIBLIOGRÁFICAS}

1 - AGRAWAL, M. P., 1966, On a new nematode Procamallanus mulleri $\mathrm{n}$. sp. from the stomach of a freshwater fish Heteropneustes fossilis. Proc. Helm. Soc. Wash., 33 (2): 204-208, 5 figs.

2 - ALI, S. M., 1960, On two new species of Procamallanus Baylis, 1923 from India with a key to the species. Indian $J$. Helminth., 34 (1/2): 129-138, 10 figs.

3 - ASCHKIN, V. M. SOBOLEV, A. A. \& KHROMOVA, L. A., 1971, Camallanata dos animais e do homem e doenças causadas por eles. In SKRJABIN, K. I., 1971. Princípios de nematodologia XXII, 388 pp., 209 figs. Akad. Nauk. S.S.S.R. ed. Moscou (em russo).

4 - CAMPANA-ROUGET, Y., 1961, Nematodes des poissons. Publ. Inst. Roy. Sci. Natur. Belgique, 4 (4): 1-57.

5 - CAMPANA-ROUGET, Y., RAZARIHELISSOA, M., 1965, Spirocamallanus olseni n. sp. (Nematoda, Camallanidae), parasite de poissons de mer de Nossi-Bé. Ann Parasit. hum Comp. 40 (2): 171-176, fig. 1; A-B, fig. 2; A-B.

6 - CAMPANA-ROUGET, Y., THEREZIEN, Y., 1965, Un noveau Procamallanus (Nematoda, Camallanidae) chez une Anguille de Madagascar. Ann. Parasit. hum. Comp., 40 (2): 165-170, fig. 1 ; A-B-C, fig. 2; fig. 3, A-B.

7 - FERNANDO, C. H., FURTADO, J. I., 1963, A study of some helminth parasites of freshwater fishes in Ceylon. Z. Parasitenk., 23, (2): 141-163, 4 pls.

8 - OLSEN, L. S., 1952, Some nematodes parasitic in marine fishes. Publ. Inst. Mar. Sci. Univ. Tex, II (2): 173-215, 84 figs.

9 - PANDE, B. P., BHATIA, B. B., RAI, P., 1963, On the Camallanid genus Procamallanus Baylis, 1923 , in two of the freshwater fishes. Indian. $J$. Helminth., 15 (2): 105-118, 17 figs.

10 - PEREIRA, C., 1935, Ascaridata e Spirurata parasitos de peixes do Nordeste Brasileiro. Arch. Inst. Biol., 6 (6): 53-62, 25 figs.

11 - PEREIRA, C., DIAS, M. V., \& AZEVEDO, P., 1936, Biologia do nematóide Procamallanus cearensis 1. sp. Arch. Inst. Biol., 7 (17): 209-226, 8 figs., 4 est.

12 - PINTO, R. M., NORONHA, D., 1972, Redescrição de Procamallanus inopinatus Travassos, Artigas \& Pereira, 1928 (Nematoda, Camallanoidea) Atas. Soc. Biol. Rio de Janeiro, 15 (2): 105-108, 10 figs.

13 - PINTO, R. M., FERNANDES, B. M. M., 1972, Sobre Procamallanus probus n. sp. (Nematoda, Camallanoidea) Atas Soc. Biol. Rio de Janeiro, 15 (3): 133-136, 6 figs.

14 - SAHAY, U., 1966, On a new nematode of the genus Spirocamallanus (Camallanidae, Procamallaninae, Nematoda) with a review of the genus. Ind. J. Helminth., 18 (2): 114-122, 4 figs.

15 - SOOD, M. L., 1967, On some species of the genus Procamallanus Baylis, 1923 from freshwater fishes of India. Proc. Nat. Acad. Sci. India, 37 (B) 1II: 291-303, 6 pl. 22 figs.

16 - TORNQUIST, N., 1931, Die Nematoden familien Cucullanidae und Camallanidae nebst. wertere Beitragen zur Kenntnis der Anatomie und Histologie der Nematoden Goteborgis Vetensk., Samih Hanse (5B) 2:1-141, 17 taf.

17 - TRAVASSOS, L.; ARTIGAS, P.; PEREIRA, C., 1928, Fauna helmintológica dos peixes de água doce do Brasil. Arch. Inst. Biol., 1:5-68 est. $1-14,155$ figs.

18 - VAZ, Z.; PEREIRA, C., 1934, Con tribuição ao conhecimento dos nematóides de peixes fluviais do Brasil. Arch. Inst. Biol São Paulo, 5: 37-103, 36 figs.

19 - VICENTE, J. J.; SANTOS, E., 1972, Sobre um novo nematódeo camallanídeo, parasito de peixe marinho (Nematoda, Camallanoidea) $A$ tas Soc. Biol. Rio de Janeiro, 15 (13): 145-147, 5 figs.

20 - YAMAGUTI, S., 1961, Systema Helmin thum 3. The nematodes of vertebrates Parte I: 1-679, Parte II: 681-917, 1125-1261, 102 pls, 909 figs. Interscience Publishers Inc. ed New York.

21 - YORKE, W.; MAPLESTONE, P. A., 1926, The nematode parasites of vertebrates VII $+536 \mathrm{pp}$. 307 figs. J. \& A. Churchill ed. London. 


\section{ESTAMPA I}

\section{Procamallanus (Spirocamallanus) pimelodus sp. $n$.}

Fig. 1 - Cápsula bucal do parátipo (Col. Helm. I.O.C. 30.999 b). Vista lateral.

Fig. 2 - Cápsula bucal do holótipo macho. Vista lateral.

Fig. 3 - Corte da cápsula bucal do pará tipo (Col. Helm. I.O.C. 30.999 d). Vista frontal.

\section{Procamallanus (Spirocamallanus) \\ intermedius sp. $\mathrm{n}$.}

Fig. 4 - Cápsula bucal do parátipo (Col. Helm. I.O.C. 31.022 c). Vista lateral.

\section{Procamallanus (Spirocamallanus) rarus}

Travassos, Artigas \& Pereira, 1928.

Fig. 5 - Cápsula bucal do parátipo (Col. Helm. I.O.C. 31.026 b). Vista lateral. (Originais). 
Mem. Inst. Oswaldo Cruz

$72(3 / 4): 205 / 211-1974$
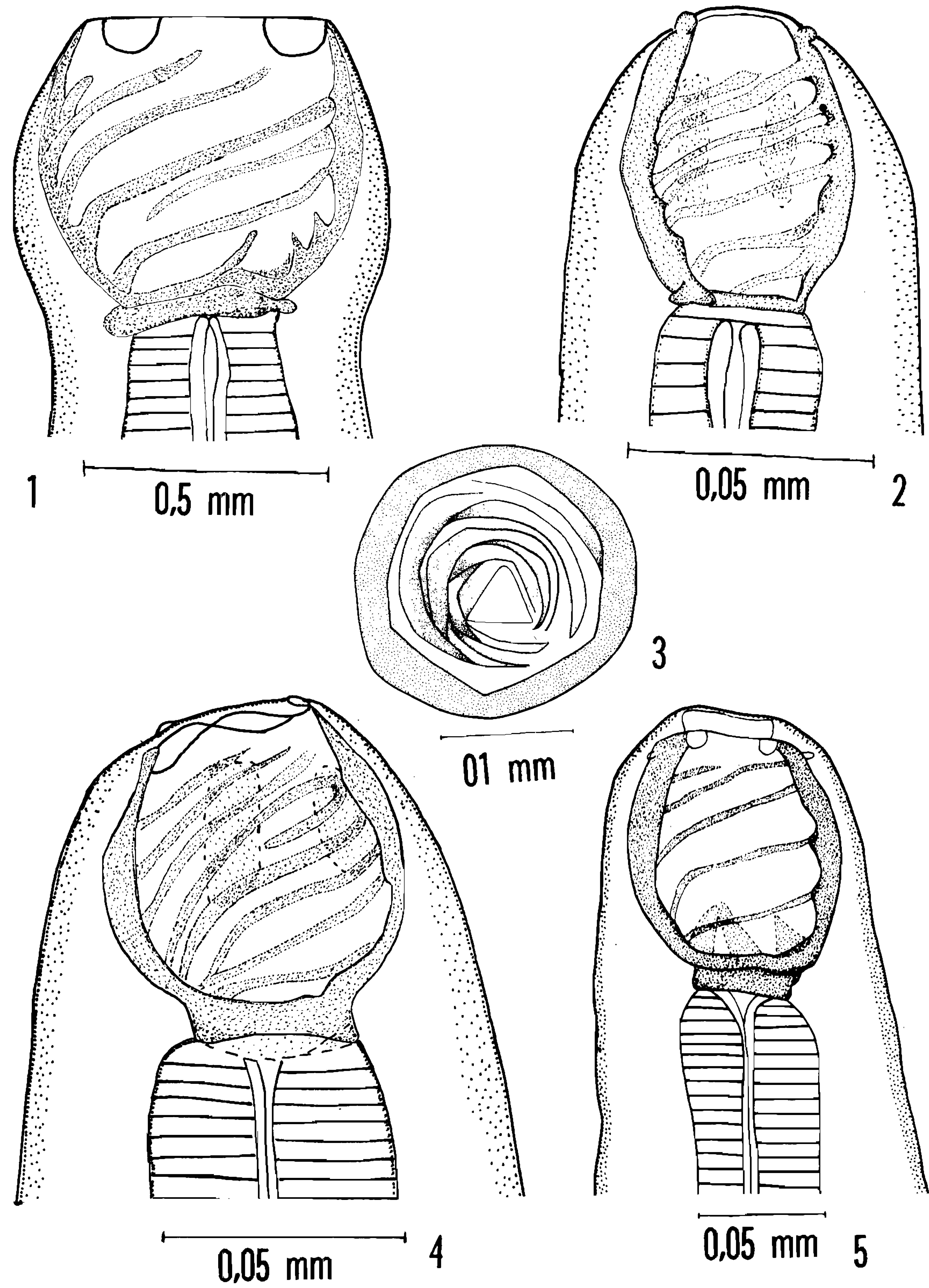

1
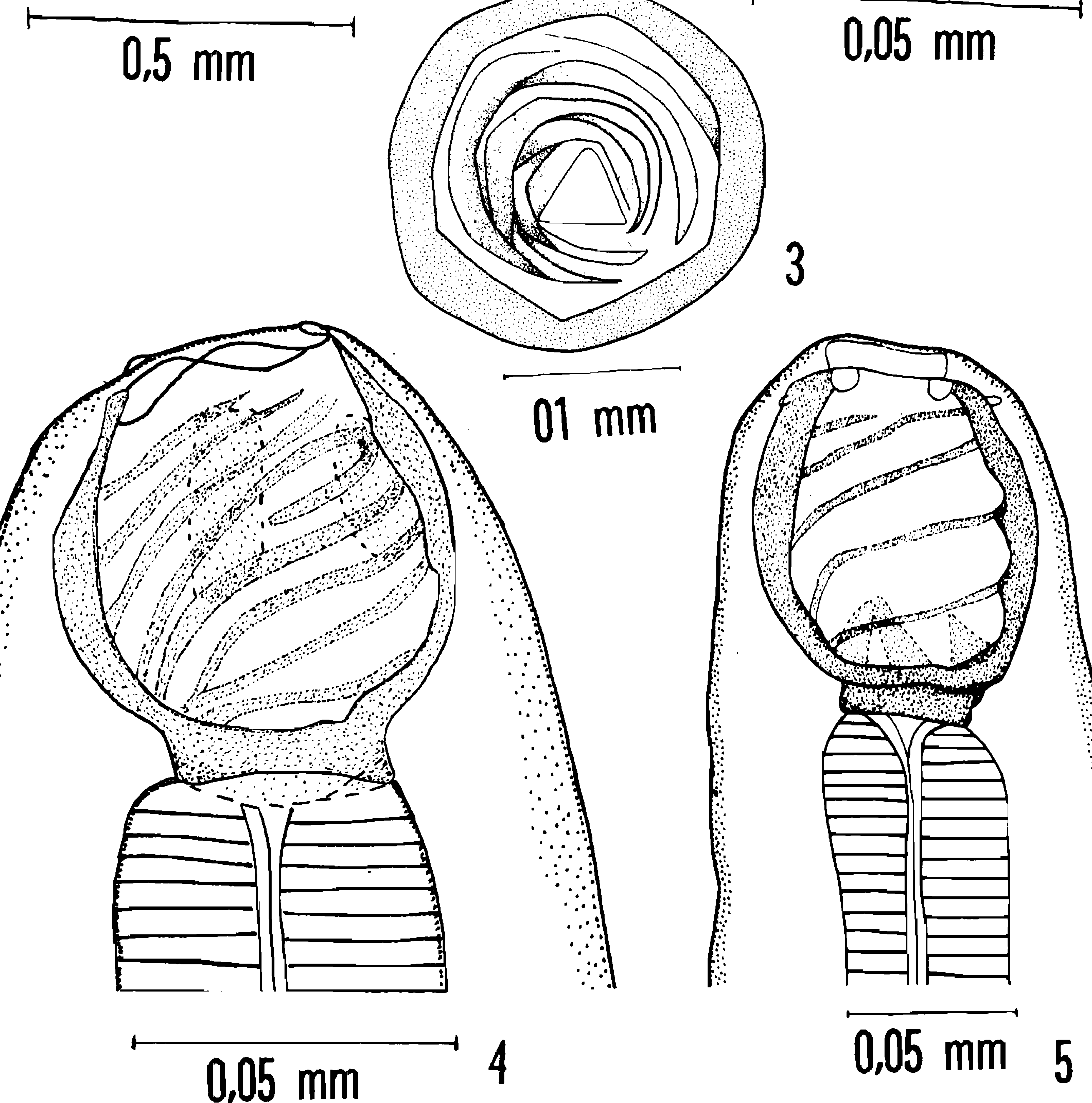

Pinto, Fabio, Noronha \& Rolas: Procamallanus 


\section{ESTAMPA II}

\section{Procamallanus (Spirocamallanus) \\ intermedius $\mathrm{sp} . \mathrm{n}$.}

Fig. 6 - Microfotografia da cápsula bucal do parátipo (Col. Helm. I.O.C. 31.022 e). Vista lateral. (7 X 40).

\section{Procamallanus (Spirocamallanus) \\ pimelodus sp. $\mathrm{n}$.}

Fig. 7 - Microfotografia da cápsula bucal do parátipo (Col. Helm. I.O.C. 30.999 b). Vista lateral. (7 X 40).

\section{Procamallanus (Spirocamallanus) rarus}

Travassos, Artigas \& Pereira, 1928.

Fig. 8 - Microfotografia da cápsula bucal do parátipo (Col. Helm. I.O.C. 31.026 b). Vista lateral. (7 X 40). (Originais). 
$72(3 / 4): 205 / 211$ - 1974

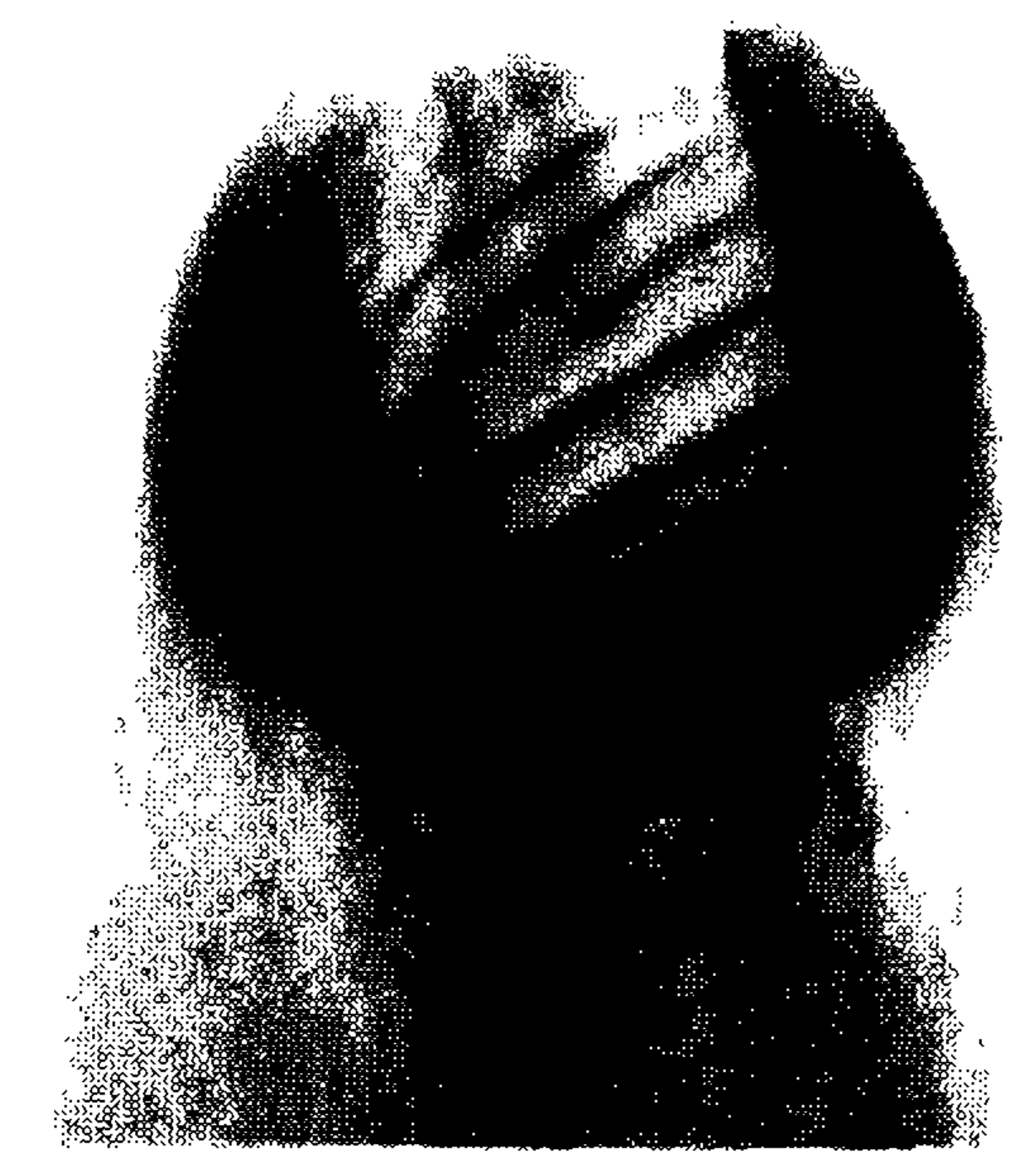

6

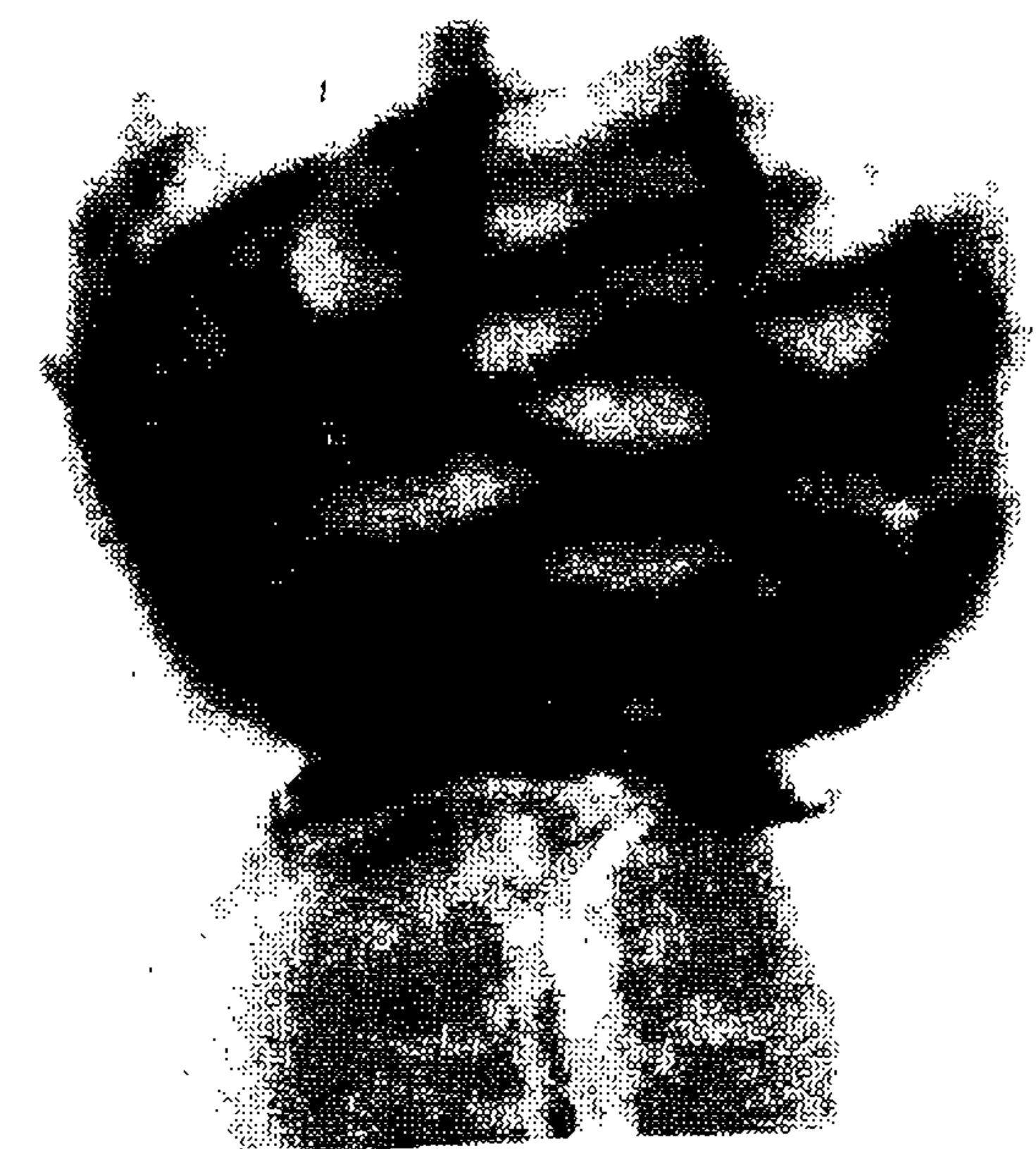

7

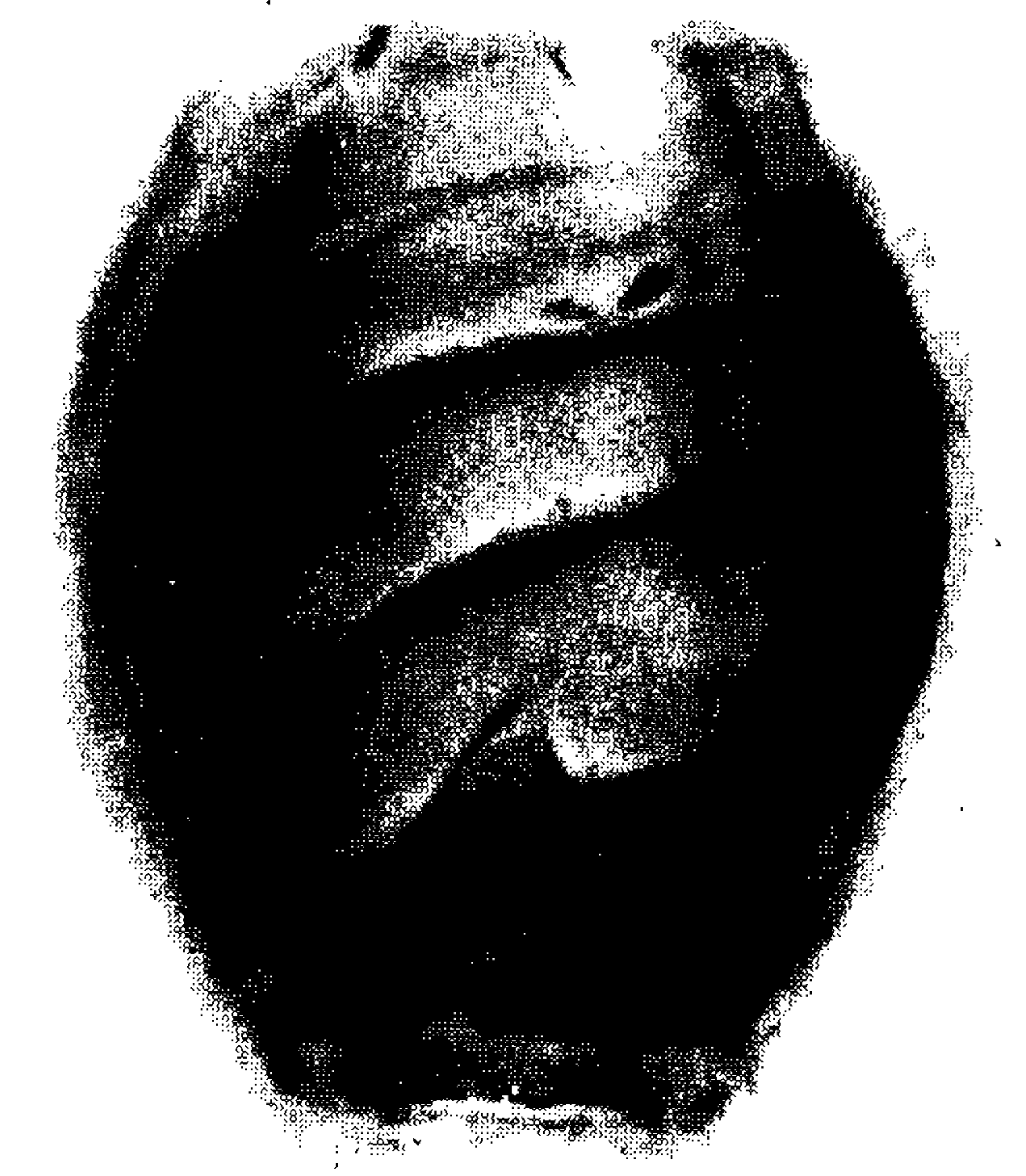

8 


\section{ESTAMPA III}

\section{Procamallanus (Spirocamallanus) pimelodus sp. $\mathrm{n}$.}

Fig. 9 - Extremidade posterior do parátipo (Col. Helm. I.O.C. 30.995 a). Vista frontal. Fig. 10 - Ovejetor do holótipo fêmea.

Fig. 11 - Extremidade posterior do holótipo macho. Vista lateral.

Fig. 12 - Reto do parátipo (Col. Helm. I.O.C. 30.997 a). Vista lateral.

Fig. 13 - Extremidade anterior do parátipo (Col. Helm. I.O.C. 30.998 b). (Originais). 
Mem. Inst. Oswaldo Cruz

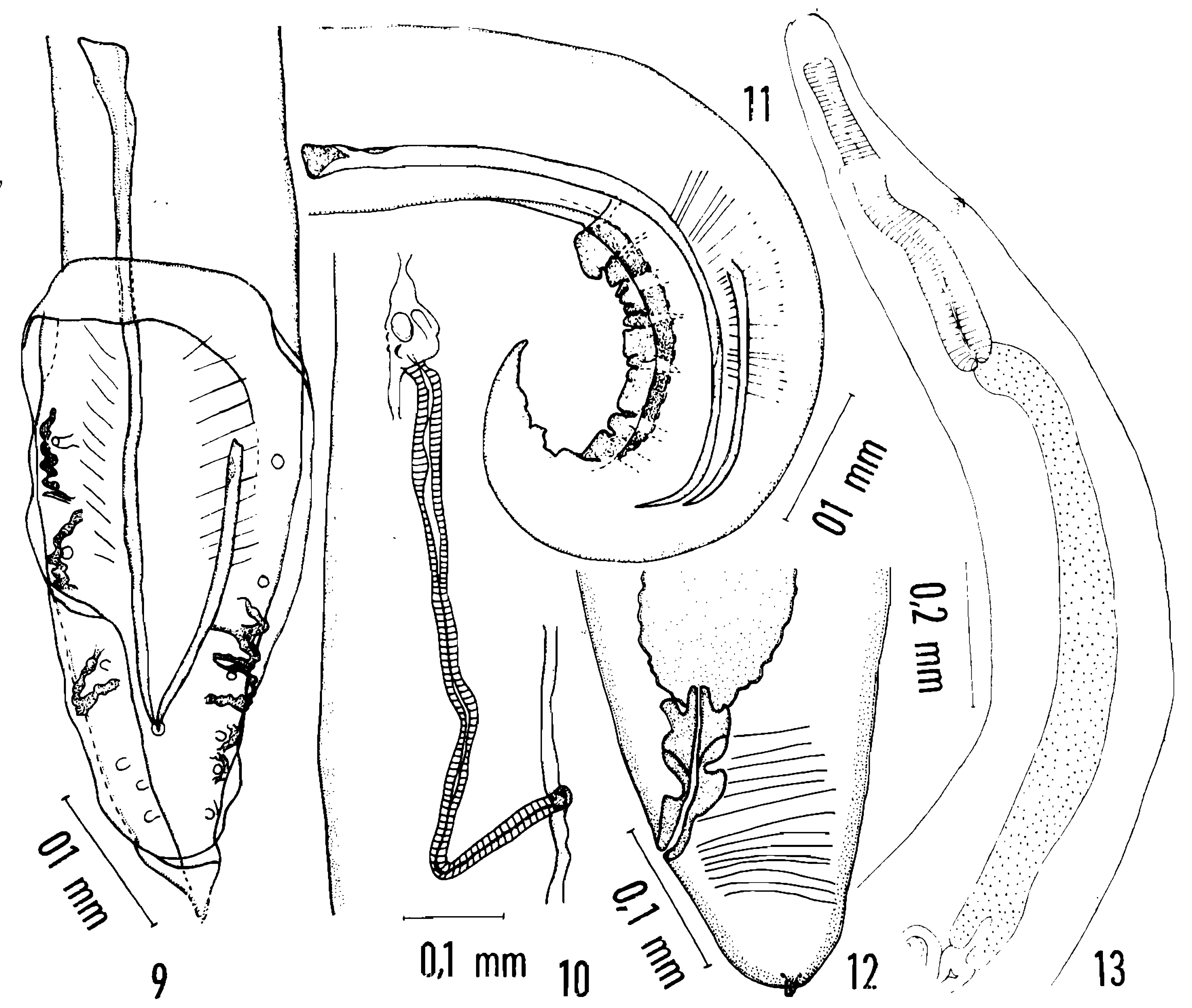


ESTAMPA IV

\section{Procamallanus (Spirocamallanus) \\ intermedius sp. $\mathrm{n}$.}

Fig. 14 - Extremidade anterior do holótipo macho.

Fig. 15 - Reto do holótipo fêmea.

Fig. 16 - Extremidade posterior do holótipo macho. Vista semifrontal.

Fig. 17 - Região distal do espículo maior do holótipo macho. Vista frontal. (Originais). 


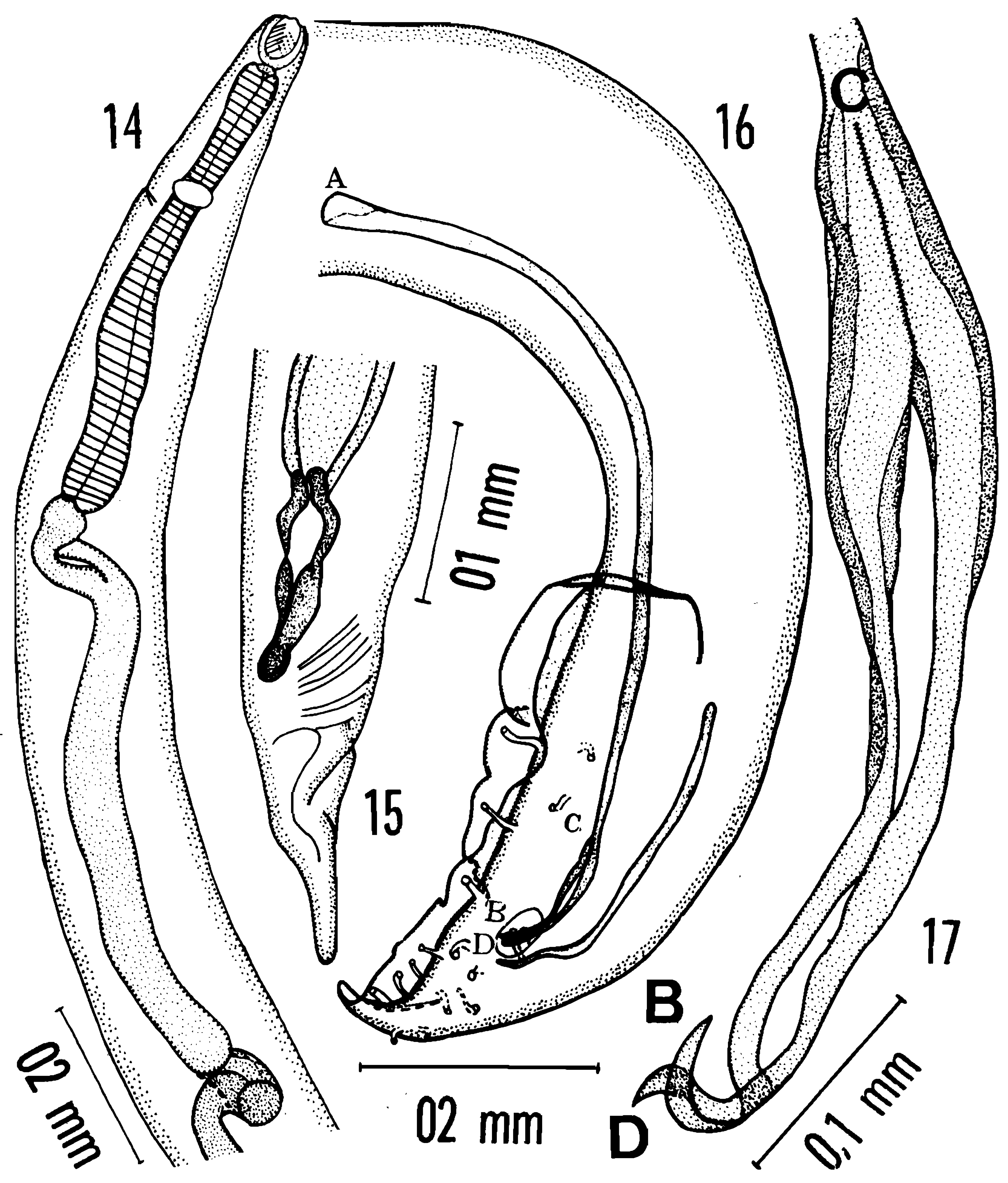




\section{ESTAMPA V}

\section{Procamallanus (Spirocamallanus) rarus}

Travassos, Artigas \& Pereira, 1928.

Fig. 18 - Extremidade anterior do parátipo (Col. Helm. I.O.C. 31.026 b).

Fig. 19 - Extremidade posterior do parátipo (Col. Helm. I.O.C. 31.028 b). Vista lateral.

Fig. 20 - Região distal do espículo maior do parátipo (Col. Helm. I.O.C. 31.028 b).

Fig. 21 - Reto do parátipo (Col. Helm. I.O.C. 31.027 b).

Fig. 22 - Espículos do tipo (Col. Helm. I.O.C. 31.029). (Originais). 


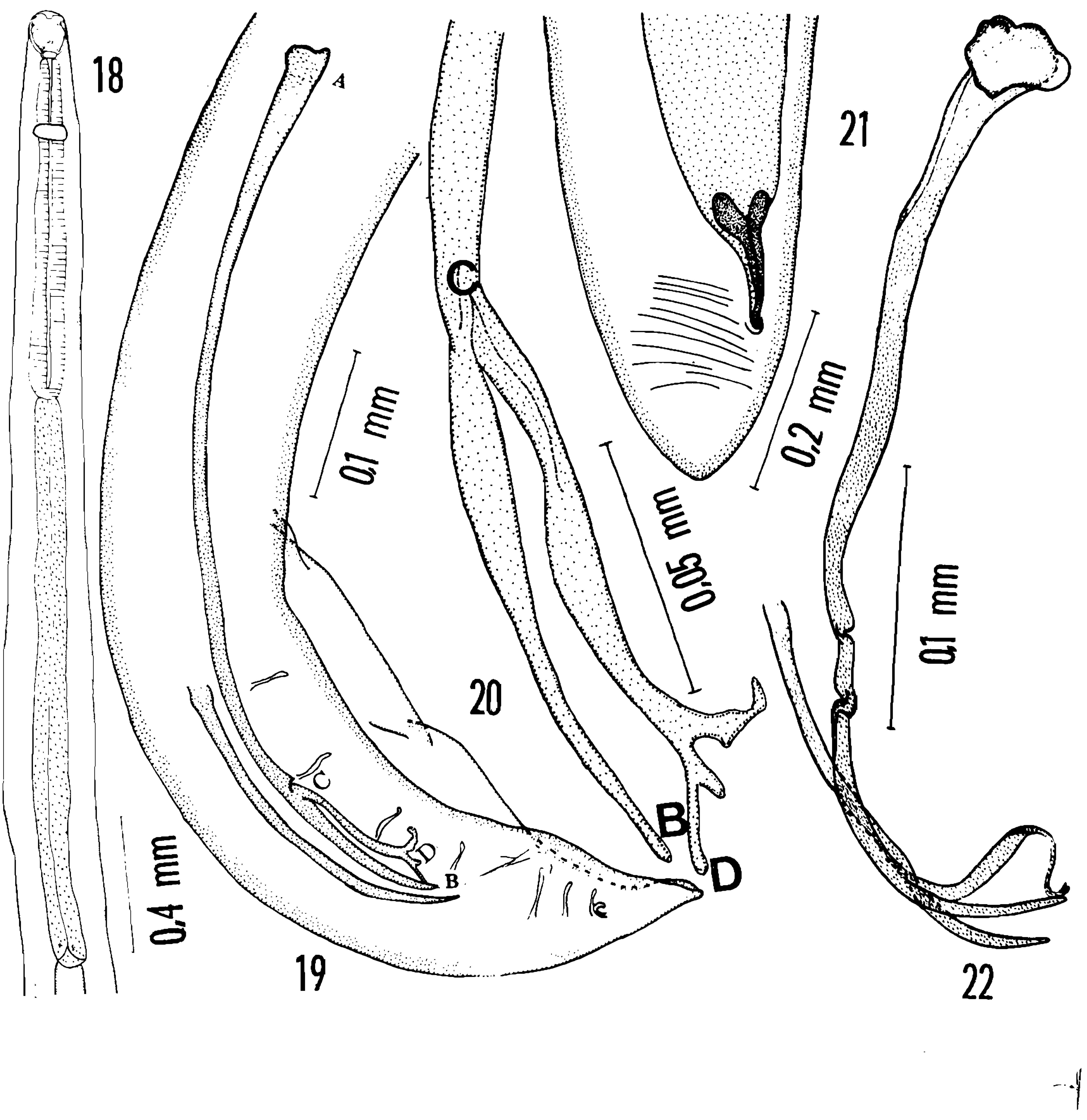

